\title{
ORIGINAL
}

\section{DIFERENCIAS DE GÉNERO EN LA PERCEPCIÓN DEL LOGRO PROFESIONAL EN ESPECIALISTAS DE MEDICINA FAMILIAR Y COMUNITARIA (*)}

\section{Lorena Saletti-Cuesta (1), Ana Delgado (1), Teresa Ortiz- Gómez (2) y Luis Andrés López- Fernández (1).}

(1) Escuela Andaluza de Salud Pública.

(2) Departamento de Historia de la ciencia y Anatomía Patológica. Facultad de Medicina. Universidad de Granada.

(*) Ayudas concedidas por el Fondo de Investigación Sanitaria (Expediente PI071176) y los Fondos FEDER de la Unión Europea.

Sin conflicto de intereses.

\begin{abstract}
RESUMEN
Fundamentos: El concepto de logro es clave para estudiar el desarrollo profesional. En medicina existen desigualdades de género en la carrera profesional. El objetivo fue conocer y comparar la percepción de resultados y atribuciones de logro en médicas y médicos de familia de Andalucía.

Método: Estudio cualitativo con 12 grupos de discusión. Población: médicas y médicos de familia que trabajan en atención primaria. Muestra: intencional segmentada por edad, sexo y ocupación de la dirección del centro de salud. Realizamos por sexo: dos grupos de jóvenes, dos grupos de mayores y dos de directores/as; total 32 médicas y 33 médicos. Análisis de contenido.

Resultados: Médicas y médicos perciben de igual modo los logros internos y coinciden en considerar como logros externos aspectos inherentes a la profesión. La diferencia más importante es que las médicas relacionan el logro con los vínculos afectivos y los médicos con méritos institucionales. Para las médicas las atribuciones internas son más importantes y destacan la importancia de la familia, la organización de la jornada laboral y el balance familia-trabajo. Para los médicos las atribuciones más importantes son sus pacientes, la formación continuada, los recursos disponibles y el sistema informático.
\end{abstract}

Conclusiones: Existen similitudes y diferencias entre médicas y médicos en la percepción de logro. Las diferencias se explican por el sistema de género. La percepción de logro de las médicas cuestiona la cultura profesional e incorpora nuevos valores. Las atribuciones reflejan el impacto desigual de variables familiares y organizacionales y apunta a que las médicas estarían modificando rasgos de la socialización de género.

Palabras clave: Sexismo.Género.Carrera.Éxito. Médicos de atención primaria.

\section{Correspondencia}

Ana Delgado

Campus Universitario de Cartuja

C/Cuesta del Observatorio 4

18080 Granada. España

ana.delgado.easp@juntadeandalucia.es

\section{ABSTRACT \\ Gender Differences in the Perception of Professional Achievement in Family Medicine, Spain}

Background: The concept of achievement is important to study the professional development. In medicine there are gender inequalities in career. The purpose was to know and compare the professional achievement's perceptions and attributions of female and male primary care physicians in Andalusia.

Method: Qualitative study with 12 focus groups (October 2009 to November 2010). Population: primary care physicians. Sample: intentionally segmented by age, sex and health care management. Were conducted by sex: two groups with young physicians, two groups with middle aged and two with health care management. Total: 32 female physician and 33 male physicians. Qualitative content analysis with Nuddist Vivo.

Results: Female and male physicians agree to perceive internal achievements and to consider aspects inherent to the profession as external achievements. The most important difference is that female physician related professional achievement with affective bond and male physician with institutional merit. Internal attributions are more important for female physician who also highlight the importance of family, the organization of working time and work-family balance. Patients, continuing education, institutional resources and computer system are the most important attributions for male physician.

Conclusions: There are similarities and differences between female and male physicians both in the understanding and the attributions of achievement. The differences are explained by the gender system. The perception of achievement of the female physicians questions the dominant professional culture and incorporates new values in defining achievement. The attributions reflect the unequal impact of family and organizational variables and suggest that the female physicians would be changing gender socialization. care.

Keyword: Sexism. Gender. Career. Achievement. Physicians, primary 


\section{INTRODUCCIÓN}

El logro profesional se define como la suma de resultados positivos del trabajo y de la vida personal relacionados con la experiencia profesional de una persona en cualquier momento de su carrera ${ }^{1}$. Es un concepto social y dinámico que se construye en un contexto histórico y cultural ${ }^{2} \mathrm{y}$, por tanto, influido por el sistema de género. El logro es clave para el desarrollo profesional y tiene dos componentes: objetivo y subjetivo o percibido. El logro objetivo se refiere a los estándares profesionales compartidos, definidos de manera externa al sujeto y considerados tradicionalmente sinónimos de éxito, por ejemplo la investigación o el desempeño de cargos ${ }^{1-3}$. Se ha demostrado que las médicas de diferentes países obtienen menos logros objetivos que los médicos ${ }^{4-6}$. El logro percibido es multidimensional y más complejo que el objetivo ${ }^{7}$, consiste en la autoevaluación de los propios logros según estándares y aspiraciones personales e incluye aspectos de satisfacción, reconocimiento y autoeficacia ${ }^{1-3}$.

En la percepción del logro se identifican dos dimensiones: los resultados esperados, es decir, la concepción personal de logro, que afecta a las decisiones profesionales y personales $^{8}$ y las atribuciones causales o factores que explican la obtención o no de $\operatorname{logros}^{9,10}$. Las atribuciones son claves para la motivación al logro, ya que las expectativas de alcanzar un logro dependen, en gran medida, de las causas a las que se han atribuido los mismos en experiencias anteriores ${ }^{9}$. Los resultados esperados del logro difieren según el género. Para las mujeres, los logros profesionales son más personales e independientes del reconocimiento externo ${ }^{11-13}$ y se relacionan con la necesidad de afiliación y con el balance entre diversas esferas de la vida ${ }^{8,11,14}$. Para los hombres, en cambio, los logros predominantes son el protagonismo personal y el reconocimiento social $^{12,14-16}$.
Algunos estudios sugieren que las mujeres atribuyen sus logros a causas externas (apoyo, azar, etc.) y sus fracasos a factores internos (habilidad, esfuerzo, etc), mientras que los hombres hacen lo contrario ${ }^{13,17-19}$.

Elaboramos un marco de referencia para investigar la percepción de logro profesional en medicina de familia en dos dimensiones: resultados esperados del logro, internos (o de gratificación personal) y externos (asociados al reconocimiento y a las características del trabajo), y atribuciones causales internas y externas.

El objetivo del estudio es conocer si existen diferencias en la percepción de resultados y atribuciones de logro en médicas y médicos de familia que trabajan en Andalucía.

\section{SUJETOS Y MÉTODOS}

Diseño. Investigación cualitativa mediante grupos focales. La población de estudio fue la de las médicas y los médicos de familia que ejercían en los centros de salud de capitales andaluzas. Mediante muestreo intencional a partir del listado de profesionales y apoyo de informantes clave, se seleccionó una muestra segmentada por edad (igual o menor y mayor de 40 años), sexo y ocupación de la dirección del centro. Los criterios de diversidad muestral fueron la formación de postgrado en medicina familiar y comunitaria, ser tutor/a de residentes y la situación familiar (tabla 1).

En 2009 y 2010 se realizaron 12 grupos de duración media de 90 minutos, con una coordinadora y una observadora. Se solicitó el consentimiento para grabar y trascribir la sesión. Participaron 65 profesionales $(5,4$ de media por grupo). El guión de la entrevista grupal (anexo 1) fue elaborado a partir de la bibliografía y de investigaciones previas del equipo y sometido a una prueba de jueces. 
Tabla 1

Características de las médicas y los médicos participantes en los grupos de discusión

\begin{tabular}{|l|c|c|}
\hline & Médicas $\mathrm{n}=32$ & Médicos $\mathrm{n}=33$ \\
\hline Edad & & \\
\hline$\leq 40$ años & 10 & 25 \\
\hline$>40$ años & 22 & \\
\hline Dirección centro de salud & & 8 \\
\hline Sí & 9 & 25 \\
\hline No & 23 & \\
\hline Especialidad MIRMF & 25 & 18 \\
\hline Sí & 7 & 15 \\
\hline No & & 14 \\
\hline Tutor/a de residentes & 17 & 19 \\
\hline Sí & 15 & 2 \\
\hline No & & 23 \\
\hline Situación familiar & 5 & 5 \\
\hline Vive sola/o con o sin hijos/as & 20 & 2 \\
\hline Vive en pareja con hijos/as & 4 & 1 \\
\hline Vive en pareja sin hijos/as & 3 & \\
\hline Otras situaciones & 0 & \\
\hline No contesta & & \\
\hline
\end{tabular}

Realizamos el análisis de contenido en tres fases: 1) Codificación: Lectura literal de las transcripciones para descomponer el texto separándolo en unidades de registro y posteriormente codificación de los párrafos en categorías predefinidas (según los objetivos del estudio) y emergentes. 2) Triangulación de categorías: Tras la doble lectura en profundidad de la trascripción de los grupos y de la primera codificación, consensuamos las categorías de análisis según los objetivos planteados y redefinimos las categorías definitivas (anexo 2). 3) Análisis pragmático: síntesis y agrupación de los datos obtenidos interrelacionando las categorías con los perfiles de los grupos ${ }^{20}$. Utilizamos el programa Nudist-Vivo8.

\section{RESULTADOS}

\section{Resultados esperados de logro profesional}

1) La satisfacción laboral es un resultado interno del logro común a todos los perfiles (figura 1).
Médicas y médicos expresan que la satisfacción del logro es interna (tabla 2, A y B) y perciben como un logro disfrutar con el trabajo (tabla 2, C y D). El trabajo bien hecho (tabla 2, E y F), la superación de retos personales, la afiliación y la utilidad social del trabajo (tabla 2, G) son resultados internos considerados menos importantes por profesionales jóvenes de ambos sexos.

Las médicas señalan que la satisfacción del logro es independiente de los méritos establecidos por la administración sanitaria (tabla 2, H).

2) Se observan desigualdades de género en los resultados externos del logro (figura 2)

Hubo elevado consenso en valorar como logros los siguientes aspectos de la vida profesional: la estabilidad laboral (plaza en propiedad), mantener buen nivel formativo, investigar, el reconocimiento de superiores y la actividad asistencial, que incluye la relación con pacientes, las actividades diagnósticas y las actividades comunitarias (tabla $3, \mathrm{~A}, \mathrm{~B}$ y C). 
Figura 1

Frecuencia de aparición de las categorías de resultado interno del logro en los relatos

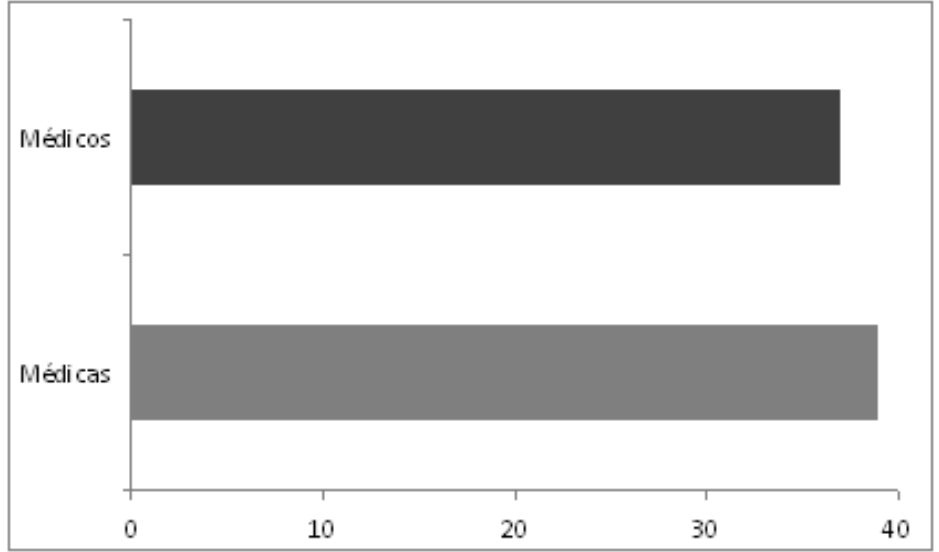

Figura 2

Frecuencia de aparición de las categorías de resultados externos del logro en los relatos

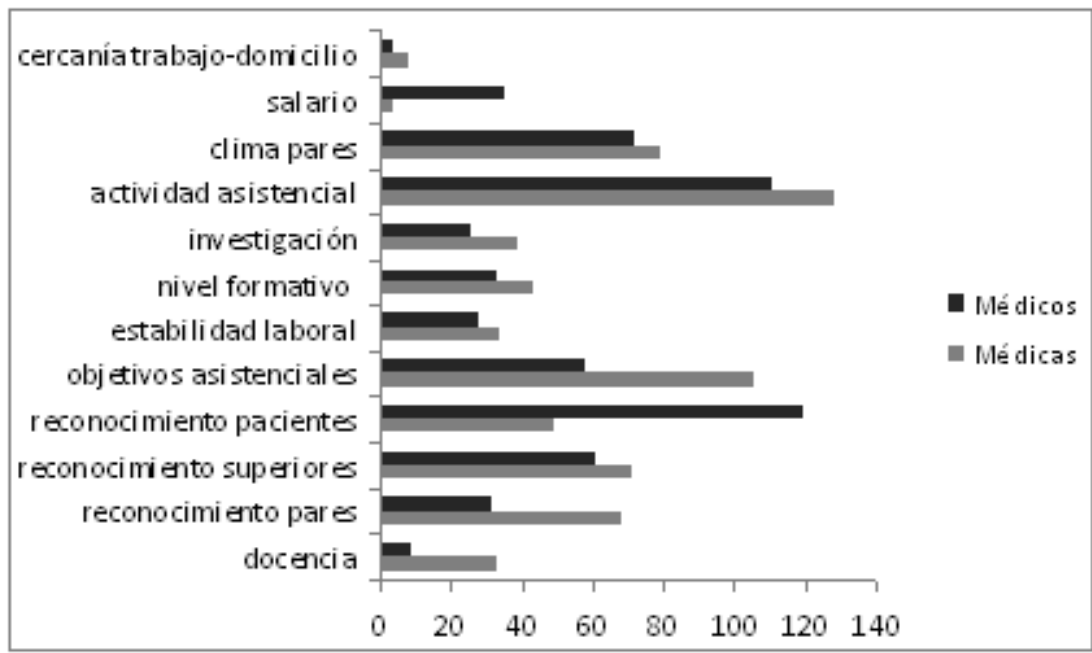


Tabla 2

\section{Citas textuales sobre los resultados internos de logro profesional}

\begin{tabular}{|c|c|}
\hline \multirow[t]{2}{*}{ Satisfacción interna del logro } & $\begin{array}{l}\text { A: Yo entiendo el éxito ¿me entiendes? como estar contento } \\
\text { conmigo mismo, es decir con haber cumplido mis expectativas } \\
\text { y yo me he marcado unas expectativas que yo entiendo como } \\
\text { cumplibles es decir no he sido, no he pecado a lo mejor de fal- } \\
\text { ta de ambición pero tampoco he sido tan ambicioso como para } \\
\text { marcarme unas expectativas inalcanzables con lo cual siempre } \\
\text { me van a hacer estar descontento ¿me entiendes? de la situa- } \\
\text { ción, pues para mi el éxito puede ser estar contento conmigo } \\
\text { mismo porque he alcanzado mis expectativas que puedo enten- } \\
\text { der yo como razonables y cumplibles... (grupo 4: Directores) }\end{array}$ \\
\hline & $\begin{array}{l}\text { B: Para mi el éxito sería acabar mi carrera profesional conten- } \\
\text { ta. Ese seria mi éxito [risas] Éxito de ganar mucho dinero o te- } \\
\text { ner reconocimiento, me importa bastante poco. A mi el éxito de } \\
\text { los demás no me importa, me importaría yo como persona } \\
\text { cuando termine mi carrera profesional y me jubile, decir: olé } \\
\text { tus narices que bien lo has hecho, ahora a divertirte, ese seria } \\
\text { mi éxito, lo demás me da igual (grupo 5: Médicas mayores) }\end{array}$ \\
\hline \multirow{2}{*}{ Disfrutar con el trabajo } & $\begin{array}{l}\text { C: Disfrutar, venir no solamente a trabajar ¿no? sino cuando } \\
\text { acabe el día y cuando llega el día siguiente decir tengo ganas } \\
\text { de ir a mi centro y de hacer las cosas. (grupo 4: Directores) }\end{array}$ \\
\hline & $\begin{array}{l}\text { D: Yo quiero disfrutar el trabajo, yo sé que todo el tiempo no se } \\
\text { puede, pero disfrutarlo, para mí eso ya es mucho... (grupo 10: } \\
\text { Médicas jóvenes). }\end{array}$ \\
\hline \multirow{3}{*}{ Trabajo bien hecho, y utilidad del trabajo } & $\begin{array}{l}\text { E: A mi me hace feliz estar con mis pacientes, y hacerlo bien } \\
\text { con mis pacientes... (grupo 6: Médicas mayores) }\end{array}$ \\
\hline & $\begin{array}{l}\text { F: Pero si cada mañana tú haces bien, tú crees que haces bien } \\
\text { tu trabajo, te hará feliz aunque no te lo reconozcan. Si al final } \\
\text { de año o dentro de cinco años te lo reconocen o te dan una ca- } \\
\text { ja de mantecados mejor [risas]No quita que mañana me levan- } \\
\text { te con la misma ilusión que no haya habido ninguna pegatina, } \\
\text { ninguna. (grupo 8: Médicos mayores) }\end{array}$ \\
\hline & $\begin{array}{l}\text { G: Ser capaz de cambiar actitudes de la gente, ser capaz de } \\
\text { ayudar a que alguien deje de fumar o a que alguien se controle } \\
\text { una diabetes o a que alguien deje de vivir la tristeza como una } \\
\text { depresión...el todavía tener cierto resorte para ser capaz de } \\
\text { ayudar (...) a que la gente cambie determinadas actitudes o } \\
\text { comportamientos me sigue pareciendo un logro casi mági- } \\
\text { co...(grupo 4: Directores) }\end{array}$ \\
\hline Logro independiente de los méritos establecidos & $\begin{array}{l}\text { H: ... a mí lo que realmente me llena es que esté satisfecha con } \\
\text { lo que hago y que mis pacientes estén contentos con lo que yo } \\
\text { hago, eso, para mí, es logro profesional. Es decir, la carrera } \\
\text { profesional tal como está establecida, a mí personalmente (...) } \\
\text { a lo mejor yo soy un bicho raro, pues no me está produciendo } \\
\text { la satisfacción que yo teóricamente en un momento de mi vida } \\
\text { me habia planteado: pues yo sería la mujer feliz del mundo } \\
\text { cuando hiciera esto, esto, esto y esto. Pues no, pues mire usted, } \\
\text { pues no. (Grupo 6: médicas mayores) }\end{array}$ \\
\hline
\end{tabular}


Tabla 3

Citas textuales sobre los resultados externos de logro profesional

\begin{tabular}{|c|c|}
\hline \multirow{3}{*}{$\begin{array}{l}\text { Estabilidad, nivel } \\
\text { formativo y actividad } \\
\text { asistencial. }\end{array}$} & $\begin{array}{l}\text { A: Yo creo que a corto plazo un logro profesional simplemente es estar en un si- } \\
\text { tio con un poco de estabilidad y poder tener un seguimiento de los pacientes, } \\
\text { que al fin y al cabo eso es la medicina de familia (grupo 9: Médicas jóvenes) }\end{array}$ \\
\hline & $\begin{array}{l}\text { B: Para mi, mi logro como médico de familia es conseguir un nivel formativo, } \\
\text { continuo, permanente que garantice que la asistencia que presto sea una asis- } \\
\text { tencia de calidad. (grupo 3: Directores) }\end{array}$ \\
\hline & $\begin{array}{l}\text { C: Pues mira, hoy si he conseguido solucionar una cosa o he dado en el clavo con } \\
\text { un asunto o realmente has tenido una buena comunicación con el paciente y dices } \\
\text { pues mira hoy ha salido algo que está en condiciones...(grupo 11: Médicos jóvenes) }\end{array}$ \\
\hline \multirow{2}{*}{$\begin{array}{l}\text { Reconocimiento de } \\
\text { pacientes }\end{array}$} & $\begin{array}{l}\text { D: Que el que entre por la puerta opine lo que quiera vamos, a mí eso me da, yo lo } \\
\text { que busco es a nivel personal y si después los pacientes me quieren y me adoran } \\
\text { bien pero sino me da igual, haciendo las cosas bien. (grupo 11: Médicos jóvenes) }\end{array}$ \\
\hline & $\begin{array}{l}\text { E: A mí lo que más satisfacción me da de mi profesión, quizá es lo que tú sientes que } \\
\text { haces por los demás y lo que notas que ellos también reciben. El tema de la afectividad } \\
\text { me gusta mucho en nuestra profesión, el que tú quieras a tus pacientes y ellos también } \\
\text { te quieren y estás valorada, y te echan de menos...(grupo 6: Médicas mayores) }\end{array}$ \\
\hline Ser docente & $\begin{array}{l}\text { F: Para mí un logro también es la tutoría, que te reconozcan, o que te terminen di- } \\
\text { ciendo: muchas gracias, he resuelto esto con lo que aprendí de ti. Eso también es un } \\
\text { logro personal para mí. Que haya sido útil en ese sentido de iniciar buenas ilusio- } \\
\text { nes, buenas prácticas en alguien que está contigo. (grupo 2: Directoras) }\end{array}$ \\
\hline Salario & $\begin{array}{l}\text { G: Creo que el logro sería una realización personal en la parte científico-técni- } \\
\text { ca, en la parte económica y en la parte promocional de la profesión... En el as- } \\
\text { pecto de realización personal y económica, que también es importante porque } \\
\text { aqui todos tenemos hijos y tenemos familia... (grupo 7: Médicos mayores) }\end{array}$ \\
\hline \multirow{3}{*}{ Clima entre pares } & $\begin{array}{l}\text { H: Que haya un ambiente en el centro de salud agradable también es muy importan- } \\
\text { te ¿no? luego el trato, el día el día con los compañeros (grupo 10: Médicas jóvenes) }\end{array}$ \\
\hline & $\begin{array}{l}\text { I: Es un logro para mí, y está más en mis manos, mejorar la gestión de personal, } \\
\text { mejorar el clima organizacional del centro, hacer que la gente entienda de una } \\
\text { forma más adecuada los conflictos y las explosiones que se producen todos los } \\
\text { días en el sistema sanitario, con lo que nos piden con lo que podemos dar, con } \\
\text { los medios que tenemos, con lo que nos exigen con el aumento exponencial de la } \\
\text { cartera de servicios...El hacer que eso se acople al trabajo diario de cada per- } \\
\text { sona y que no explote... (grupo 3: Directores) }\end{array}$ \\
\hline & $\begin{array}{l}\text { J: Para mí ahora mismo el éxito en la gestión es tener al grupo, o sea, a los } \\
\text { compañeros bien, satisfechos, seguros y con ganas de trabajar, con ilusión... } \\
\text { que no haya rencillas, que no haya problemas...que la gente venga a hacer las } \\
\text { cosas con ganas...conseguir la unión... eso es... jun éxito! (grupo 2: Directoras) }\end{array}$ \\
\hline \multirow[t]{2}{*}{$\begin{array}{l}\text { Cumplimiento de los } \\
\text { objetivos del contrato } \\
\text { programa }\end{array}$} & $\begin{array}{l}\text { K: Que la población de mi unidad esté mejor atendida y que ellos estén conten- } \\
\text { tos y que el centro yo vea que tiene una dinámica de grupo, que funcione, que si } \\
\text { además tenemos buenos indicadores de preinscripción, pues divinamente, pero } \\
\text { para mi eso no es el objetivo principal. Por eso te digo que cuando voy al Dis- } \\
\text { trito lo único que me preguntan es por los objetivos, presupuesto, objetivos, ca- } \\
\text { pitulo uno... Y casi nadie me pregunta: Oye y cómo van los indicadores de va- } \\
\text { cuna, o cómo te has reunido con está gente, o... eso me decepciona a mi un } \\
\text { poco... (grupo 2: Directoras) }\end{array}$ \\
\hline & $\begin{array}{l}\text { L: cumplir con los logros del sistema porque siempre es agradable también ver } \\
\text { como tú colaboras un poco en aquello que te has marcado como objetivo ¿no? y } \\
\text { eso a mí por lo menos me gusta ¿no? saber que estoy dentro de una línea y aun- } \\
\text { que hay cosas que no comparta pero en otras pues sí, me gusta estar en la línea } \\
\text { de lo que se está primando ¿no?... (grupo 4: Directores) }\end{array}$ \\
\hline
\end{tabular}


Figura 3

Frecuencia de aparición de las categorías de atribuciones internas del logro en los relatos

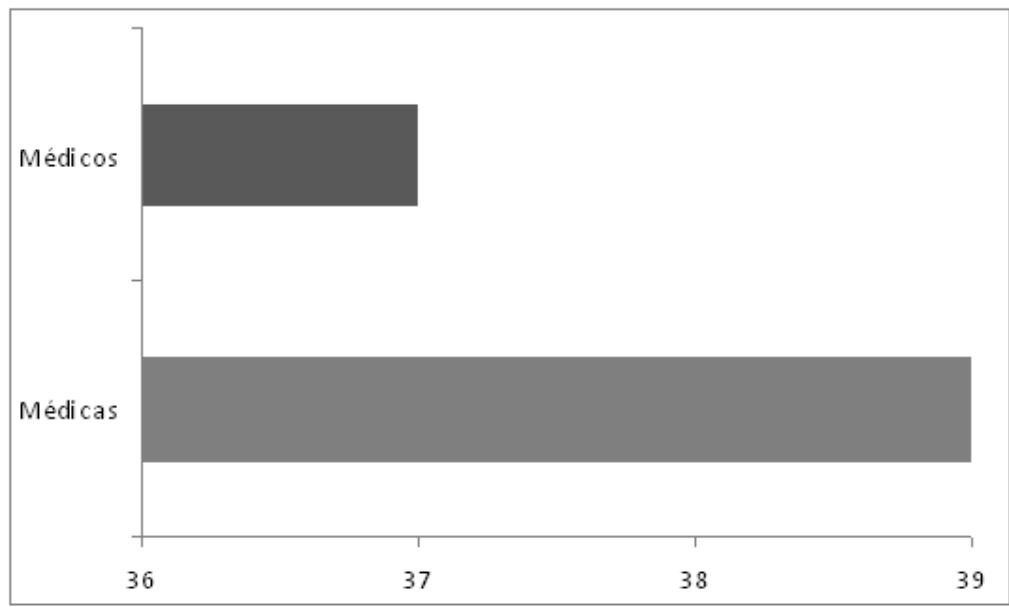

Todos los grupos mencionaron la importancia de obtener el reconocimiento de sus pacientes. Los médicos varones debatieron más que las médicas, como se observa en la figura 2, sobre el significado de este reconocimiento y antepusieron su satisfacción profesional (tabla 3, D). Para las médicas, el reconocimiento de sus pacientes significa per se un vínculo afectivo (tabla 3, E), siendo difícil de alcanzar para las jóvenes a causa de la inestabilidad laboral.

La cercanía del trabajo al domicilio y el reconocimiento y apoyo de fueron pares son logros más importantes para las médicas de todos los perfiles que para los médicos. Para las mayores la docencia fue fuente de reconocimiento, satisfacción y estímulo profesional (tabla 3, F). El salario fue más mencionado como un logro profesional por los médicos que por las médicas (tabla 3, G).

Desempeñar la dirección de un centro de salud fue considerado un logro tanto por las directoras como por los directores. Unas y otros valoraron el buen clima entre pares más que el resto de grupos. $\mathrm{Al}$ ocupar un rol jerárquico, diferente al res- to de integrantes del equipo, directoras y directores se sienten responsables del clima entre pares (tabla 3, H). Los directores lo consideraron un medio necesario para el trabajo (tabla 3, I) y las directoras lo entendían como un objetivo en sí mismo, en ocasiones arduo de alcanzar (tabla 3 , J). Las directoras debatieron más en el grupo sobre el cumplimiento de los objetivos del contrato programa que, para la mayoría, no constituía un logro (tabla 3 , $\mathrm{K})$. Para los directores, por el contrario, sí lo era, debido al reconocimiento institucional que puede reportar (tabla 3, L).

\section{Atribuciones del logro profesional}

En los discursos grupales, las atribuciones causales se identificaron como factores cuya presencia o ausencia actúa, respectivamente, de estímulo o barrera para el logro.

1) Las atribuciones internas para alcanzar el logro son más importantes para las médicas (figura 3)

La personalidad, las expectativas propias, la experiencia, la motivación y el control de situaciones y sentimientos son 


\section{Tabla 4}

\section{Citas textuales sobre las atribuciones internas del logro}

\begin{tabular}{|l|l|}
\hline \multirow{5}{*}{$\begin{array}{l}\text { Personalidad, expectativas, } \\
\text { experiencia, motivación, } \\
\text { control de situaciones } \\
\text { y sentimientos }\end{array}$} & $\begin{array}{l}\text { A: Tu personalidad, tus habilidades de comunicación, capacidad de liderazgo.... } \\
\text { Todo eso pues influye que consigas determinados logros ino? sobre todo pen- } \\
\text { sando en el aspecto de dirección, y la experiencia. (grupo 2: Directoras) }\end{array}$ \\
\cline { 2 - 3 } & $\begin{array}{l}\text { B: A míme dificulta muchas veces mi propia personalidad, a veces pienso que no } \\
\text { voy a ser capaz de, de, de, de dar la talla ¿no? de hacer las cosas como a mí me } \\
\text { gusupo 5: Médicas mayores) }\end{array}$ \\
\cline { 2 - 3 } & $\begin{array}{l}\text { C: Depende muchísimo de cómo se encuentre uno, de su estado emocional. Si uno } \\
\text { está pasando una situación personal jodida, por muy bien que esté el trabajo, et } \\
\text { trabajo no lo ve bien o no te sientes con logros o no te sientes con éxitos. Entonces, } \\
\text { depende muchisimo de las caracteristicas personales de cada uno y las vivencias } \\
\text { que tenga cada uno en cada momento. (grupo 7: Médicos mayores) }\end{array}$ \\
\hline
\end{tabular}

características personales, o atribuciones internas, relacionadas con la consecución de un logro. Todas las médicas, fundamentalmente las de los grupos de mayores, percibían que son importantes porque pueden contribuir de forma positiva o negativa a alcanzar sus logros (tabla 4, A y B). Por el contrario, eran pocos los médicos que vincularon el logro con atribuciones internas (tabla $4, \mathrm{C}$ ).
2) Existen desigualdades de género en las atribuciones externas del logro (figura 4)

La relación con pares (tabla 5, A y B) y ser docente con residente a su cargo (tabla 5, C y D) fueron factores para alcanzar logros en todos los grupos. A su vez, fueron valorados como obstáculos el escaso tiempo por paciente (tabla 5, E), la falta de

Figura 4

\section{Frecuencia de aparición de las categorías de atribuciones externas del logro} en los relatos

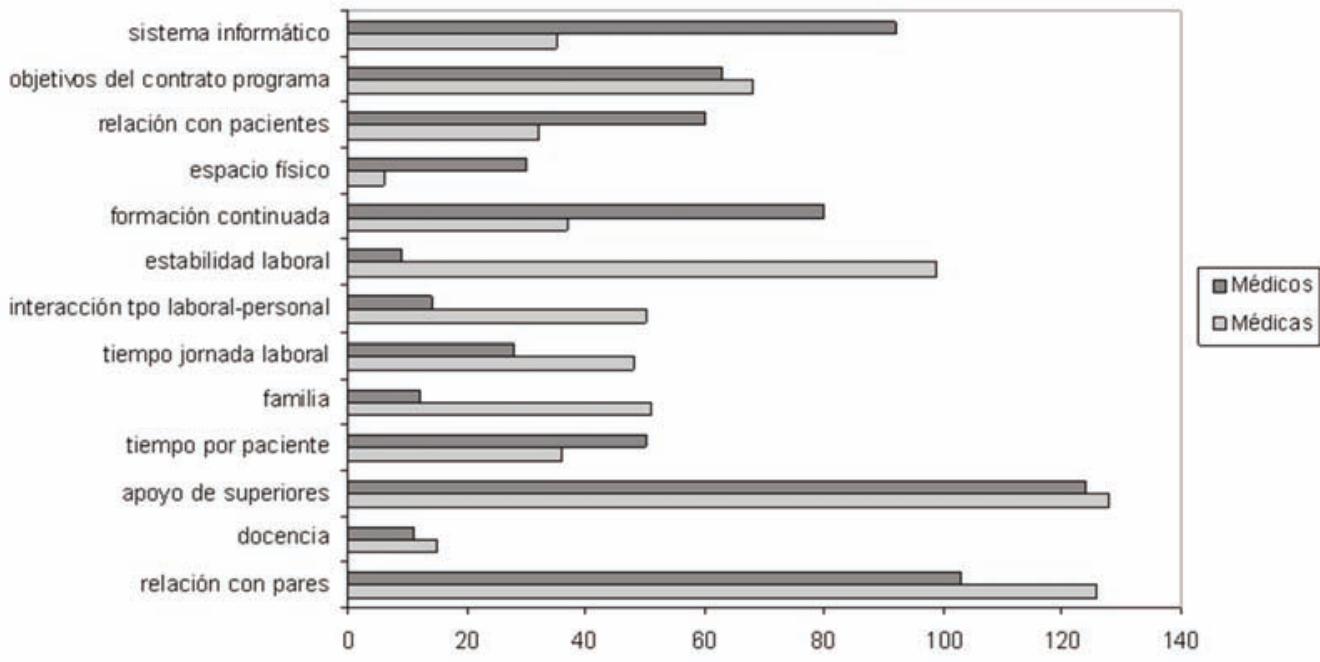




\section{Tabla 5}

\section{Citas textuales de atribuciones externas del logro}

\begin{tabular}{|c|c|}
\hline \multirow{2}{*}{ Relación con pares } & $\begin{array}{l}\text { A: Otra cosa que te facilita conseguir cosas es el equipo, contar con un buen equipo, tú pue- } \\
\text { des hacer muchas más cosas que si tienes piedras en el camino...Si todo el mundo tira para } \\
\text { alante y todo el mundo se prepara su sesión estupendamente y todo el mundo tiene ilusión } \\
\text { por hacerla, la cosa es completamente distinta... (grupo 5: Médicas mayores) }\end{array}$ \\
\hline & $\begin{array}{l}\text { B: El hecho de trabajar en equipo, el hecho de trabajar en el centro de salud donde hay va- } \\
\text { rios médicos en contacto, con sesiones clínicas o cuando estás tomándote el cafelillo "hoy he } \\
\text { tenido un tio que tiene esto, esto y esto, ¿qué será, no?" "Ah pues yo tuve uno que parecia es- } \\
\text { to". Eso hace que tú vayas progresando en tu profesión...facilita mucho que tu vayas mejo- } \\
\text { rando (grupo 12: Médicos jóvenes) }\end{array}$ \\
\hline \multirow[b]{2}{*}{$\begin{array}{l}\text { Ser docente con residente } \\
\text { a su cargo }\end{array}$} & $\begin{array}{l}\text { C: Para mi también es un logro y un reto cada año con un residente nuevo... Entonces conse- } \\
\text { guir que el residente... darte cuenta de dónde has podido quedarte corto o haber fallado, que } \\
\text { el del año siguiente todaví, si puede ser, salga mejor formado (grupo 8: Médicos mayores) }\end{array}$ \\
\hline & $\begin{array}{l}\text { D: En un centro con residentes las sesiones van mucho mejor... iy te ayuda un montón! bueno y } \\
\text { si tienes residentes más todavia, yo por lo menos he tenido durante seis meses una residente y } \\
\text { claro te obliga porque claro estás súper motivada ¿no? entonces te obliga también a poder, pa- } \\
\text { ra poder darle yo qué sé ¿no?, lo que tú puedes aportarle estar lo más actualizada posible ¿no? } \\
\text { a la hora de cualquier cosa ¿no? a lo mejor no sé, hacer las cosas mejor también ¿no? (grupo } \\
\text { 10: Médicas jóvenes) }\end{array}$ \\
\hline Tiempo por paciente & $\begin{array}{l}\text { E: Mi logro sería intentar llegar más a la gente ¿no? ¿De qué carezco para poder llegar a la } \\
\text { gente? Pues fundamentalmente como ahora mismo es la falta de tiempo, es decir tengo muy } \\
\text { poco tiempo por paciente y me cuesta dificilmente poder abordar una problemática en cinco } \\
\text { o seis minutos. (grupo 6: Médicas mayores) }\end{array}$ \\
\hline \multirow{3}{*}{ Apoyo de superiores } & $\begin{array}{l}\text { F: Quiero que me dejen hacer las cosas bien, es más, yo he discutido con muchos directores de } \\
\text { centros de salud, con muchos, y a todos les he dicho lo mismo “'tú te crees que es digno que } \\
\text { me pongas ochenta pacientes hoy y los avisos de dos cupos?” Eso no es digno, ¿no? (grupo 9: } \\
\text { Médicas jóvenes) }\end{array}$ \\
\hline & $\begin{array}{l}\text { G: Yo creo que un condicionante fundamental es sentirte apoyado y respaldado por tus } \\
\text { jefes me parece un condicionante, yo he vivido experiencias en ambos sentidos. Ha ha- } \\
\text { bido un momento en que me he sentido muy respaldado y ha habido un momento en que } \\
\text { no me he sentido respaldado y creo que ha sido uno de los mayores condicionantes para } \\
\text { conseguir o no conseguir algo... (grupo 4: Directores) }\end{array}$ \\
\hline & $\begin{array}{l}\text { H: Facilitador sería que a los directores le adecuasen el cupo asistencial... quien te ha } \\
\text { nombrado sí debería organizar y estudiar tu carga asistencial. Eso no se ha hecho, no se } \\
\text { hace...no hay una actuación, una intervención activa de quien te nombra, diga: "bueno, } \\
\text { pues yo quiero que tú seas director de un centro, que te ocupes de este centro y para ello } \\
\text { te voy a estudiar tu carga laboral, tu carga de trabajo, tu consulta y tu agenda. No: tú } \\
\text { organizate, hazte la agenda, lleva tu cupo. (grupo 3: Directores) }\end{array}$ \\
\hline \multirow{2}{*}{$\begin{array}{l}\text { Objetivos del contrato } \\
\text { programa }\end{array}$} & $\begin{array}{l}\text { I: A mí si me afecta mucho porque eso en el tiempo que yo he estado más tiempo en un cupo, } \\
\text { que me he esforzado mucho por llevar la consulta, por sacarlo todo adelante, pues luego eso } \\
\text { en las reuniones solo se hablaba de indicadores y a lo mejor otros aspectos de tu trabajo se } \\
\text { quedan tapados ¿no? parece que todo va en lo que has recetado, en las derivaciones que has } \\
\text { hecho, si te pasas ya no eres buen médico, que yo creo que no tiene ninguna relación, hombre } \\
\text { hay que controlarlo, seremos gestores también ino? tenemos que controlarlo un poco pero } \\
\text { hay muchos más aspectos que a lo mejor no se valoran, solamente las cifras... (grupo 10: Mé- } \\
\text { dicas jóvenes) }\end{array}$ \\
\hline & $\begin{array}{l}\text { J: ... Lo que veo es que los que mandan, los que dan los mandatos lo hacen tan mal que } \\
\text { nos hacen quedar mal, porque a la gente le están diciendo una cosa "vaya usted a su mé- } \\
\text { dico, que su médico lo manda donde usted diga y donde a usted le haga falta" y a nos- } \\
\text { otros nos están diciendo "mira, no los mandes, manda menos y mándale cosas más ba- } \\
\text { ratas", entonces ese doble mensaje al final lo sufres tú en tu trabajo habitual y eso hace } \\
\text { que tú lo pases mal en tu trabajo y es una barrera... (grupo 10: Médicos jóvenes) }\end{array}$ \\
\hline
\end{tabular}




\section{Tabla 5 \\ Citas textuales de atribuciones externas del logro (continuación)}

\begin{tabular}{|c|c|}
\hline \multirow{3}{*}{ Relación con sus pacientes } & $\begin{array}{l}\text { K: A mí me han ayudado mis pacientes a conseguir logros ... O sea me ha dolido } \\
\text { cuando un paciente me ha hecho una crítica y además llevaba toda la razón pero a } \\
\text { mí si me ha ayudado ¿eh? a conseguir logros en el sentido de mejorar la comuni- } \\
\text { cación. (grupo 1: Directoras) }\end{array}$ \\
\hline & $\begin{array}{l}\text { L: Al final te acomodas tú a los pacientes y los pacientes a ti y no terminas de re- } \\
\text { solver las cosas como tendrías que hacerlas... a lo mejor que veas a alguien en la } \\
\text { lista ya está aquí otra vez, ya se me han acabado los recursos para intentar solu- } \\
\text { cionar las cosas. (grupo 12: Médicos jóvenes) }\end{array}$ \\
\hline & $\begin{array}{l}\text { M: Yo creo que hay ahí una desventaja importante que es ser mujer y joven, mujer jo- } \\
\text { ven es mezcla explosiva, eres la niña. (grupo 9: Medicas jóvenes) }\end{array}$ \\
\hline \multirow[b]{2}{*}{ Familia } & $\begin{array}{l}\text { N: Otra cosa que me ha facilitado mucho llegar a conseguir lo que quería creo que } \\
\text { ha sido mi familia también, porque siempre me han estado empujando para delante, } \\
\text { para que yo pudiera hacer lo que, lo que no me creía capaz de hacer y sin embargo } \\
\text { ellos siempre han estado ahí impulsándome ¿no? (grupo 5: Médicas mayores) }\end{array}$ \\
\hline & $\begin{array}{l}\text { O: Y otra cosa que nos limita mucho porque es verdad que cuando tienes niños pe- } \\
\text { queños te limitan tus niños pequeños pero cuando ya tienes una determinada edad y } \\
\text { tienes los padres también en edad de empezar a enfermar te limitan grandemente, es } \\
\text { decir que bueno, que eso también, es decir que los dos extremos de la vida por ser } \\
\text { mujer, quizá no lo sé porque por la educación que nos han dado o por la situación en } \\
\text { la que hemos vivido, pues ahora tenemos eh pasamos de ser cuidadoras de nuestros } \\
\text { hijos a ser cuidadoras e nuestros padres ¿no? (grupo 6: Médicas mayores) }\end{array}$ \\
\hline Tiempo jornada laboral & $\begin{array}{l}\text { P: Si no hubiera la demanda que hay y tuviéramos este rato para estudiar como lo } \\
\text { hacen en otros países, ya no sé yo, bueno... en el norte por lo menos ya lo hacen } \\
\text { así en algunos hospitales, pero aqui es que tienes que sacar de tu tiempo libre, y } \\
\text { entonces eso si nos agota... (grupo 5: Médicas mayores) }\end{array}$ \\
\hline Interacción tiempo laboral- tiempo personal & $\begin{array}{l}\text { Q: A mí me gustaría tener tiempo y que ese tiempo no fuera a costa de la salud per- } \\
\text { sonal mía ni de la salud de mi familia, es decir que cuando he tenido que sacar tiem- } \\
\text { po he tenido que sacrificarme yo y sacrificar a mi familia...es decir no dedicarle el } \\
\text { tiempo suficiente que mi familia ha necesitado de mi por echarle tiempo al trabajo } \\
\text { (grupo 6: Médicas mayores) }\end{array}$ \\
\hline Estabilidad laboral & $\begin{array}{l}\text { R: Con contratos de verdad [risas] pues te dan todos esos derechos ¿no? En los } \\
\text { contratos eventuales, los sesenta por ciento, los parciales, todo esto, pues siempre } \\
\text { tienen la trampa para que no tengas esos derechos, entonces eso es lo que te difi- } \\
\text { culta tener esa conciliación... No sabes cuando te van a llamar, si te van a llamar, } \\
\text { si dices que no que es lo que pasa después, porque tienes contratos cada tres me- } \\
\text { ses o cada dos meses y eso te hace muy dificil conciliar ivamos!... (grupo 10: Mé- } \\
\text { dicas jóvenes) }\end{array}$ \\
\hline Formación continuada & $\begin{array}{l}\text { S: Los cursos que se imparten desde Distrito, desde otro tipo de entidad a mí me } \\
\text { agrada enormemente que no sean los laboratorios los que nos llevan de la mano a } \\
\text { dar cursos sino que sean instituciones neutrales... (grupo 12: Médicos jóvenes) }\end{array}$ \\
\hline Sistema informático & $\begin{array}{l}\text { T: Intentar dentro de lo posible que nos afecte lo menos posible evidentemente el } \\
\text { día que no funciona el sistema informático no funciona y punto y ya está, para in- } \\
\text { tentar que te afecte lo menos posible a ti y a ti en la relación con el paciente por- } \\
\text { que muchas veces ya en vez de relación médico-paciente es la relación médico-pa- } \\
\text { ciente-ordenador porque se mete dentro de la relación también ¿no? (grupo } 12 \text { : } \\
\text { Médicos jóvenes) }\end{array}$ \\
\hline
\end{tabular}


apoyo de superiores (tabla 5, F, G y H) y los objetivos del contrato programa, por dificultar la relación con pacientes y pares (tabla 5, I y J).

La relación con sus pacientes es importante en todos los grupos, si bien los médicos debaten más al respecto. De esta relación señalan aspectos positivos, como el aprendizaje mutuo y la confianza (tabla 5, K) y negativos, como la medicalización, y la falta de responsabilidad que conlleva en sus pacientes o las inercias asociadas al continuo trato y gran conocimiento de las personas de su cupo (tabla 5, L). La falta de respeto por parte de sus pacientes es percibida con mayor frecuencia por las médicas jóvenes, que la atribuyen precisamente a su doble condición de mujeres y jóvenes (tabla 5, M).

La familia fue considerada como apoyo (tabla $5, \mathrm{~N}$ ) y como obstáculo (tabla $5, \mathrm{O}$ ) para las médicas de todas las edades. Opinaban que la jornada laboral debería incluir tiempo para la formación, la investigación y la organización del trabajo y que para alcanzar logros es necesario un equilibrio entre el tiempo dedicado a la vida laboral y a la vida personal (tabla 5, P y Q).

Otro factor decisivo para el logro de las médicas jóvenes es la estabilidad laboral, debido a que influye directamente en la relación con pares y pacientes, en la formación, en el disfrute de derechos laborales, y en la organización de su vida personal y laboral (tabla 5, R).

Para los varones de todos los grupos los elementos más facilitadores de sus logros fueron la formación continuada (tabla 5, S) y disponer de recursos adecuados, incluido el espacio físico. Entre los obstáculos, algunos médicos jóvenes y mayores mencionaron el sistema informático, que burocratiza la consulta y dificulta la atención clínica (tabla 5, T).

\section{DISCUSIÓN}

Este trabajo aporta información relevante sobre la dimensión subjetiva del logro enprofesionales de medicina de familia enAndalucía. Los resultados muestran que tanto en la concepción personal de logro como en las atribuciones causales existen similitudes y diferencias entre mujeres y hombres y que el género juega un papel fundamental en la percepción del logro profesional.

Los discursos grupales modulan y enriquecen el marco conceptual de partida en varios aspectos. Por una parte se observa que un mismo elemento puede identificarse como logro en sí y como atribución del logro. Así ocurre con las relaciones entre pares o la relación con pacientes. Emerge también una visión más compleja de la atribución del logro, ya que un mismo factor, como por ejemplo el apoyo de superiores o la familia, puede actuar en sentido positivo y negativo.

Llama la atención que la dimensión externa, tanto en resultados de logro como en atribuciones, incluya un número de categorías muy superior al de categorías incluidas en la dimensión interna. La creación de dichas categorías emerge de los discursos grupales, y es posible que esta diferencia se deba a la existencia de dificultades de traducir al lenguaje las ideas y emociones internas y subjetivas, algo que no ocurre con la designación de los aspectos externos fácilmente identificables.

Los logros internos se perciben de igual modo en ambos sexos y la gratificación personal por el trabajo bien realizado es un valor compartido en medicina de familia en nuestro entorno. También coinciden, médicas y médicos, en definir como logros externos aspectos considerados, por otra parte, inherentes a la profesión médica, como la actividad asistencial de calidad, la formación continuada, el clima entre pares ${ }^{21}$ o tener una plaza en propiedad. 
Las desigualdades de género más relevantes aparecen en el significado asignado a algunos logros profesionales. Destaca el hecho de que los vínculos afectivos en las relaciones con pacientes y con pares y el sentimiento de satisfacción personal son más importantes para las médicas que para los médicos. Por su parte, ellos asocian el logro a los méritos institucionales o al salario. Estas mismas diferencias se han encontrado en estudios sobre distintas profesiones ${ }^{8,12,13}$.

Desde una perspectiva de género, Barberá y Cantero ${ }^{13}$ recogen diversas hipótesis para explicar estas diferencias de sexo en la definición de logro, entre ellas el miedo al éxito, las atribuciones causales, las expectativas relacionadas con el rol de género o el lugar que las metas personales desempeñan en la motivación al logro. La socialización de género es subrayada por Virginia Valian ${ }^{18}$ alegando que existen construcciones mentales y valores implícitos adquiridos en la infancia que juegan un papel central en la carrera profesional de hombres y mujeres. En el caso de ellas, el rol de género asignado se fundamenta en los cuidados y en la esfera relacional, lo que afectaría a sus percepciones, expectativas y evaluaciones del logro y dificultaría su desarrollo profesional. Por el contrario, el rol de proveedor y los valores de autonomía y poder asignados a los hombres serían características favorecedoras de su desarrollo profesional.

La teoría de la socialización es considerada reduccionista por algunas autoras ${ }^{22,23}$, debido a que no incluye el papel de la estructura de la organización ni de los modelos de carrera vigentes. María Markus ${ }^{11}$ plantea que la definición de logro que hacen las mujeres debe interpretarse como una forma de rechazo al modelo dominante, basado en la competitividad, y como un cuestionamiento de los rígidos criterios de profesionalización vigentes que exigen la imposible elección de centrarse en la vida privada o en la vida pública. Mediante el traslado al ámbito público-profesional de algunos valo- res del sistema de género como, por ejemplo, la importancia de las relaciones personales, las mujeres estarían contribuyendo a transformar la definición de logro.

Además del papel que juega la construcción de género, es importante destacar el papel que puede desempeñar la especialidad que estamos estudiando. La medicina de familia, en su formación y desempeño incorpora la atención centrada en el paciente, promueve la orientación biopsicosocial que prima el componente afectivo, frente a la orientación biomédica donde prevalece el componente instrumental ${ }^{24,25}$. Sin embargo, a pesar de recibir una formación homogénea en esos contenidos, la investigación sobre la práctica asistencial de médicos y médicas muestra que la comunicación es diferente en ambos sexos, las médicas presentan una mayor orientación psicosocial y se centran más que los médicos en los contenidos relacionales y afectivos de la atención a sus pacientes ${ }^{26,27}$, siendo conscientes de la existencia de estas diferencias $^{28}$. Desde las ciencias sociales se ha señalado que la construcción social de las relaciones de género contribuye también a explicar la relación médico-paciente ${ }^{29}$. Los médicos varones debaten más sobre el significado del reconocimiento de sus pacientes, sin embargo son más independientes de su valoración.

El análisis de las atribuciones del logro revela el mayor peso de las de origen interno en el discurso de las médicas, para quienes la motivación, el esfuerzo y el control de las emociones son fundamentales. Este hallazgo difiere de los que señalan que las mujeres relacionan los logros con causas externas, mientras que los hombres los atribuyen a factores internos ${ }^{10,17-19}$. La discrepancia puede deberse, en parte, a las diferentes metodologías, ya que algunas de estas investigaciones se basan en evaluaciones sobre el logro realizadas por terceras personas ${ }^{17,19}$ y otras estudian poblaciones de estudiantes universitarios en lugar de profesionales en activo ${ }^{10}$. 
Por otra parte, se ha señalado que el sistema de género juega un papel en las atribuciones que realizan las personas sobre sus $\operatorname{logros}^{10,17}$. La atribución del logro a causas internas guarda relación con la percepción de autoeficacia, es decir con los juicios sobre las propias capacidades ${ }^{30}$. En un estudio reciente hemos constatado que la percepción de autoeficacia es igual en médicas y médi$\cos ^{31}$. Ello parece indicar que algunos rasgos del proceso de construcción de género, como las expectativas profesionales o la autoeficacia, varían según el contexto témporo-espacial y laboral, y que las experiencias en la percepción de logro podrían estar cambiando en la actualidad entre las médicas de familia.

Entre las atribuciones externas del logro existen aspectos comunes a ambos sexos, como la influencia de sus pares y de la docencia, el tiempo por paciente, el papel del apoyo de superiores y los objetivos del contrato programa. La importancia de estos elementos en medicina de familia ha sido destacada igualmente en otros trabajos ${ }^{32-33}$.

La relación con sus pacientes es significativa para ambos sexos y predomina en los grupos de médicos jóvenes. Se ha señalado que la experiencia facilita la negociación con los pacientes ante sus demandas y conflictos $^{34}$, negociación que puede venir dificultada por la exigente demanda de sus pacientes sobre prescripciones que el médico no considera indicadas.

Encontramos atribuciones externas relevantes que son diferentes para médicas y médicos. Para las primeras, el apoyo de la familia y el equilibrio entre vida laboral y personal facilitan alcanzar logros, mientras que la dedicación a tareas familiares y las dificultades para conciliar tiempo de trabajo y tiempo personal relacionadas con la doble presencia, lo obstaculizan. El origen de las dificultades a las que se enfrentan las médicas en este ámbito se ha atribuido a la oposición entre el mandato del cuidado como principal obligación de las mujeres y la ideología profesional, construida a su vez sobre la experiencia y los valores masculinos ${ }^{35}$. En estudios previos se ha observado que en la carrera de las médicas las relaciones familiatrabajo tienen consecuencias más relevantes en la percepción de $\operatorname{logro}{ }^{36-38}$.

En una investigación cuantitativa previa encontramos que la relación familia-trabajo se asocia con la percepción de logro tanto en médicas como en médicos de familia, si bien la relación es más fuerte en las primeras (pendiente de publicar). Al indagar este mismo fenómeno con metodología cualitativa encontramos diferencias entre médicas y médicos. Es necesario señalar que los resultados obtenidos con las metodologías cuantitativas y cualitativas aportan visiones incompletas y complementarias de la realidad. El lenguaje artificial, que mide las variables de investigación a través de las preguntas de un cuestionario, usado en la metodología cuantitativa es muy diferente al lenguaje espontáneo de los grupos de discusión, donde se reproduce lo más fielmente posible el campo de connotaciones en el que se inscribe el lenguaje natural de sus participantes en un momento histórico social concreto $^{39}$. Por ello, las metodologías cualitativas permiten entender los discursos como expresiones de la propia subjetividad y comprender los significados que otorgan las personas a su experiencia.

Para los médicos varones, los factores externos más influyentes dependen en gran medida del ámbito institucional, como la formación continuada, los recursos materiales y los sistemas de información. Estos factores, estrechamente relacionados con la organización del ámbito profesional, influyen más en el logro de los hombres, que obtienen apoyos de la misma en mayor medida que las mujeres ${ }^{3,40}$. La adecuada distribución de recursos es importante para alcanzar logros, pero está condicionada por lo que se conoce como "círculos de amigos (varones)" que raramente benefician a las 
mujeres en las organizaciones científicas en general ${ }^{41}$ y a las médicas en el sistema sanitario en particular ${ }^{40}$.

La mayor fortaleza de este estudio es el análisis conjunto de las dos dimensiones del logro, los resultados y las atribuciones, y de sus relaciones con el sistema de género.

Entre sus limitaciones cabría mencionar la diferencia de edad y de sexo entre la moderadora y algunos de los grupos. Sin embargo, podemos afirmar que se consiguió un clima de confianza que favoreció la obtención de la información buscada. Hubiera sido deseable llevar a cabo un proceso de validación de los resultados con las personas que participaron en la investigación. Los hallazgos del estudio están vinculados al contexto en el que se desarrolló el estudio y no pueden generalizarse. Sería conveniente indagar otras especialidades médicas y/o comunidades autónomas en futuros estudios.

Podemos concluir que entre médicas y médicos de familia existen similitudes y diferencias en los resultados del logro y en las atribuciones causales del mismo. Los discursos de las médicas sobre los significados del logro muestran rasgos de su socialización como mujeres a la vez que cuestionan los esquemas androcéntricos vigentes y la cultura profesional dominante, e incorporan nuevos valores en la definición de logro profesional.

Las atribuciones reflejan el impacto desigual de variables familiares y organizacionales en el logro de médicas y médicos, y apuntan a que las médicas podrían estar modificando algunos rasgos del proceso de socialización de género.

Estas diferencias se traducen en desigualdades de género y tienen un impacto importante en el desarrollo profesional de las médicas. Las administraciones sanitarias deberían incorporar a su modelo de carrera un concepto integrador de logro e implementar medidas organizativas y de corresponsabilidad dirigidas a promover el desarrollo profesional en condiciones de igualdad.

\section{AGRADECIMIENTOS}

A las médicas y los médicos de familia por su participación. A la persona que realizó el informe de revisión en la prepublicación por sus interesantes sugerencias.

\section{BIBLIOGRAFÍA}

1. Arthur M, Khapova S y Wilderom C. Career success in a boundaryless career world. J Organ Behav. 2005; 26: $177-202$.

2.Dries N, Pepermans R y Carlier O. Career success: Constructing a multidimensional model. J Vocat Behav. 2008; 73: 254-67.

3.Ng T, Eby L, Sorensen K y Feldman D. Predictors of objetive and subjetive career success: a meta-analysis. Pers Psychol. 2005; 58: 367-408.

4. Levitt C, Candib L, Lent B y Howard M. Women physician and family medicine. Monograph/literature review. 1ra ed. Orlando: Wonca Working Party on Women and Family Medicine; 2008. p.195.

5 .Observatorio Salud de la Mujer. Informe salud y género 2007-2008. Mujeres y hombres en las profesiones sanitarias. 1ra ed. Madrid: Ministerio de Sanidad y Consumo; 2009. p.75.

6. Delgado A, Saletti-Cuesta L, López-Fernández LA. Et al. Gender and the professional career of primary care physicians in Andalusia (Spain). BMC Health Services Research. 2011; 11: 51.

7. Nabi G. The relationship between HRM, social support and subjetive career success among men and women. I J Manpow. 2001; 22: 457-74.

8. Dyke L y Murphy S. How we define success. A qualitative study of what matters most to women and men. Sex Roles. 2006, 55: 357-71.

9. Weiner B. Motivation from an attribution perspective and the social psychology of perceived competence. En Elliot A. Dweck (eds). Handbook of competence and motivation. 1ra ed. Nueva York: Guilford Press; 2005. p. $73-84$ 
10. Durán-Aponte E y Pujol L. Diferencias de género y área de estudio en las atribuciones causales de estudiantes universitarios. Anales de la Universidad Metropolitana. $2012 ; 12: 39-51$.

11. Markus M. Mujeres, éxito y sociedad civil. Sumisión o subversión del principio de logro. En: Benhabib S y Cornell D (eds.) Teoría feminista y teoría crítica. 1 ra ed. Valencia: Alfons El Magnanim; 1990. p. 151-168.

12. Coria C. Los laberintos del éxito. Ilusiones, pasiones y fantasmas femeninos. Barcelona: Paidos, 1993. p.155.

13. Barberá E y Cantero MJ. Motivación del logro y categorización de género. En: Garrido Gutiérrez I. Psicología de la motivación. 1ra ed. Madrid: En síntesis; 1996. p. 287-308.

14. Kalet A, Fletcher K, Ferdman D y Bickell N. Defining, navigating, and negotiationg success. The experience of Mid-career Robert Wood Johnson Clinical Scholar Women. J Gen Intern Med. 2006; 21: 920-5.

15. Garfinkel P, Bagby M, Schuller D. et al Gender differences in the practice characteristics in Ontario. Acad Psychiatry. 2004; 28: 310-20.

16. Buckley L, Sanders K, Shih M et al. Obstacles to promotion? Values of women faculty about career success and recognition. Acad Med. 2000; 75: 283-8.

17. Swim J y Sanna L. He's skilled, she's lucky: a metaanalysis of observers'attributions for women's and men's success and failures. Pers Soc Psychol Bull. 1996; 22: $507-19$

18. Valian V. Why so slow?: the advancement of women. 1ra ed. Cambridge, Mass: 1998. p.401.

19. García-Retamero R y López-Zafra E. Atribuciones causales sobre éxito y fracaso y percepción del liderazgo femenino. Estudios de Psicología 2008; 29: 273-287

20. Navarro P y Díaz C. Análisis de contenido. En: Delgado JM y Gutiérrez J. Métodos y técnicas cualitativas de investigación en ciencias sociales. Madrid: Síntesis; 1999. p. 176-224.

21.Gallo Vallejo FJ, Altisent Trota R, Díez Espino J Et al. Perfil profesional del médico de familia Aten Primaria. $1999 ; 23: 236-45$

22. Riska E. Towards gender balance: but will women physicians have an impact on medicine? Soc Ssci Med. 52: 179-87, 2001.

23. Witz A. Professions and patriarchy. 1ra ed. Londres: Routledge; 1995
24 .Little P y Payne S. Observational study of effect of patient centeredness and positive approach on outcomes of general practice consultations. BMJ 2001, 323: 908-11.

25. Bensing JM y Drinkers J. Instrumental and affective aspects of physician behaviour. Med Care 1992, 30: 283 97.

26. Delgado A. El papel del género en la relación médicopaciente. Formación Médica Continuada en Atención Primaria 1999, 6: 509-16

27. Roter D, Hall J y Aoki Y. Physician gender effects in medical communication. A meta-analytic review. JAMA. 2002; 288: 756-64

28. Delgado A, Távora Rivero A y Ortiz Gómez T. Las médicas, sus prácticas y el dilema con la feminidad. Estudios de Sociolingüística 2003, 4: 589-611.

29. LoCicero AK. Explaining excessive rates of caesareans and other childbirth interventions: contributions from contemporary theories of gender and psychosocial development. Soc Sci Med 1993, 10:1261-9.

30. Abele A y Spurk D. The longitudinal impact of selfefficacy and career goals on objetive and subjetive career success. J Vocat Behav. 2009; 74: 53-62.

31. Delgado A, Saletti-Cuesta L, López-Fernández LA. Et al. Logro profesional alcanzado y percibido de médicas y médicos de familia. VIII Congreso Iberoamericano de ciencia, tecnología y género. Curitiba, Brasil. 5 al 9 de abril 2010 .

32. Hulscher ME, Schouten LM, Grol RP y Buchan H. Determinants of success of quality improvement collaborative: what does the literature show? BMJ Qual Saf. 2013; 22:19-31.

33. Casajuana, J. Diez minutos, ¿Qué menos!. Aten Primaria. $2001 ; 27: 297-8$

34. Walter A, Chew-Graham C y Harrison S. Negotiating refusal in primary care consultations: a qualitative study. Fam Pract. 2012; 29: 488-496.

35. Morantz-Sánchez R. Sympathy and science. Women physicians in American medicine. 1ra. Ed. Nueva York Oxford University Press; 2000. p.464.

36. Verlander G. Female physicians: balancing career and family. Acad Psychiatry. 2004; 28: 331-6.

37. Shollen L, Bland C, Finstad D y Taylor A. Organizational climate and family life: How these factors affect the status of women faculty at one medical school. Acad Med. 2009; 84: 87-91. 
38. Buddeberg-Fischer B, Stamm M, Buddeberg C, Bauer G, Hämmig O, Knecht M y Klaghofer R. The impact of gender and parenthood on physicians' careers - professional and personal situation seven years after graduation. BMC Health Services Research. 2010; 10: 40.

39. Conde F. Un ensayo de articulación de las perspectivas cuantitativa y cualitativa en investigación social. REIS. 1990; 51:91-117.

40. Melamed T. Career success: The moderating effect of gender. J Vocat Behav. 1995; 47: 35-60.

41. ETAN. Informe política científica de la Unión Europea. Promover la excelencia mediante la integración de la igualdad entre géneros. Bruselas: Comisión Europea; 2001. 


\section{Anexo 1 \\ Guión de la entrevista}

1) ¿Qué es para vosotros tener logros profesionales?

2) ¿Y pensáis que es lo mismo alcanzar logros profesionales que tener éxito como médicos de familia?

3) ¿Cuándo sentís que vosotros u otro compañero tenéis éxito o alcanzáis un logro en la profesión?

Para indagar: ¿Qué aspectos de vuestra vida profesional hacen que sintáis que tenéis éxito que habéis alcanzado un logro?

4) ¿De qué factores creéis que depende que tengáis más o menos logros profesionales o éxito en vuestra profesión?

Para indagar atribuciones negativas: ¿Cuáles creéis que son las dificultades u obstáculos para alcanzar un logro profesional o un éxito? Del entorno profesional, institucional; familiares; personales.

5) ¿Qué factores os han ayudado o apoyado a lo largo de vuestra vida profesional para que alcanzar un logro o éxito como médicos de familia? 
Anexo 2

\section{Listado final de categorías}

Resultados del logro:

- Internos:

Gratificación del trabajo

Trabajo bien hecho

Superación de retos

- Externos:

Cercanía del lugar de trabajo

Dinero (remuneración económica)

Ser docente

Realizar actividades de formación

Realizar actividades de investigación

Reconocimiento de pares

Clima laboral entre pares

Reconocimiento de pacientes

Realizar actividades asistenciales

Realizar actividades comunitarias y preventivas

Realizar procedimientos técnicos (excluida la entrevista clínica)

Obtener plaza en propiedad

Reconocimiento de superiores o jefes

Desempeñar un cargo directivo

Consecución de los objetivos del contrato programa

Atribuciones para el logro

- Internas:

Personalidad

Motivación

Auto-cuidados (control de sentimientos y situaciones)

- Externas:

Suerte

Trabajar en medio urbano o rural

Realizar actividades de formación

Ser docente y tener residentes

Pacientes

Pares

Familia

Espacio físico

Tiempo de consulta por paciente

Tiempo completo dedicado a la jornada laboral

Interacción tiempo laboral- tiempo personal

Estabilidad laboral

Objetivos del contrato programa y el sistema de evaluación

Historia clínica informatizada

Detentar un cargo directivo

Tipo de relaciones con superiores 